Historic, Archive Document

Do not assume content reflects current scientific knowledge, policies, or practices. 



\section{Wholesale Prices on Garden Seed}

\begin{tabular}{|c|c|c|}
\hline ariets & Pric & \\
\hline ASPARAGUS $10 \mathrm{lbs}$ & $5 \mathrm{lbs}$ & $1 \mathrm{lb}$ \\
\hline Columbian....... & 300 & \\
\hline Wash't' Rust Pr'f & 725 & 150 \\
\hline BEETS & & \\
\hline osbs's Egyp. & 375 & \\
\hline uson Globe 650 & 375 & 100 \\
\hline rrly Eclipse.... 650 & 375 & 100 \\
\hline lood Turnip 650 & 375 & 100 \\
\hline nip 65 & 375 & 100 \\
\hline it D' R'd $^{\prime} 1000$ & 550 & 125 \\
\hline 400 & 250 & 55 \\
\hline & $3 \pi$ & 100 \\
\hline Sug. 450 & 275 & 60 \\
\hline & & \\
\hline & 37 & 85 \\
\hline $\ln 440$ & 25 & 00 \\
\hline in 450 & 27 & \\
\hline 4 & & 60 \\
\hline & & \\
\hline
\end{tabular}

\section{CABBAGE}

Goiden Acre.... 4000

EF. Flat Dutch. 1600

Ey. Jer. Takefla14 50

Copen. M'k't... 3000

Express......... 1000

Earl క Summer.16 co

All Seasons ......1600

Early All Head 1000

Wis. Hollander 4000

Danish Ba!l Fi'd 30 0]

Ea: $0 A m \mathrm{Lr}^{\prime \prime m} \mathrm{~m} d \mathrm{~s}^{\circ} 00$

Volga

Autumn Fing.. 1600

Late Flat Dutch 1500

Gio. of Enk'isel.22 50

Folland........ 22 50

Forseman...... 200

Incope ......... 6) 00

Mam. Red Rock22 50

\section{CARROT}

New Chantenay 650

Oxheart ........ 650

Er. Scarlet 패orn 5 50

Dan. Half Loug. 500

Berry's Ey. G. B. 6 £0

Large Wh. Blgi'n 450

Mastodon......... 500

Long Orange..... 500

Victoria.......... 500

CAULIFLOWER

Early snowball.

- Is. We'ther Gi't

Berrs's Late G't

\section{CELERY}

White Plume....

Gold'n S'lf Bl'nch'g

Giant Pascal....

Golden Heart...

\section{CUCUMBER}

Imp. Long Green 900

Early Cluster... 750

Early Frame.... 750

Green Prolific....750

Chi. Pickling.... 750

Wh.SpineEv'grn 750

Imp. White Spine 750

$350 \quad 75$

$350 \quad 75$

$300 \quad 65$

$275 \quad 60$

$350 \quad 75$

$250 \quad 55$

27560

$275 \quad 60$

$275 \quad 60$

1500

1500

2000

$900 \quad 190$

2000500

900200

$1600 \quad 325$

425100

425100

425100

425160

425100

425100

Variety
Cool and Crisp.... 750
Boston Pickle.... 750
New Klondike.... 550
Cumberland..... 750
Daris Perfect....10 00
Berrys Ch. Pickl'r12 50
LETTCCE

Ey. Curl'd Simp. 1150

Bl. Seeded Simp. 1150

Sel, Big Boston.. 1150

Gran? Rapids... 1150

Imp. Hanson.... 1150

Berrys Bountif' 1150

Prizehead........ 1150

Cal.Cream But'er11 50

Mas King.........11 50

Iceberg...........1150

Crisp as Ice ....... 1150

X.Y.or Fonderf'120 00

\section{JUSKMELOY}

Rocky Ford. Sel, 700

Green Nutmeg... 700

Emerald Gem.... 800

Lg. Y Cantaloupe 950

shummas Siant 900

Burrell's Gem... 950

Berry's Farorite $1 \frac{1}{2} 00$

Montreal Miarket 700

Houdoo........... 800

Ex.E. Hackens'ck 700

Gold'n Net. Gem 700

Pollock No. 25.... 950

Banana (true) ... 1000

ip Top............ 700

Osage (Milllers Cr.) \& 00

Hones Dew....... 950

Gold Lined RkJ.F10 00

B 5's Gold Prince.15 00

\section{TITERMELON}

$500 \quad 125$

\section{Angeleno B. S... 800}

AlabanaSweet... 550

Cuban Queen..... 500

Cole's Earls...... 500

E5. Hones Drip. 14 c0

Fordhook Earls.. 500

Ga. Rattlesnake.. 500

Gulden Hones.... 750

Halbert's Hones.. 650

Hungarian Hones. 800

Harris' Earliest...5 00

Irish Gres ......... 850

Kleckles Sweet... 600

Light Icing. ....... 500

Monte Cristo...... 5 C0

McI ver's Sugar.....5 00

Mam. NeltSugar 1400

New Winter......10 co

Peerless or Ice Cr. 5 co

Phinney Ex. Ex... 500

Sweetheart..... 500

Tom Tatson.... 500

Cul.G.S'd Citron 500

Red S'd Citron.- 700

Apple Pie....... . 850

Sugar Stick.... 1400

Stone Mount'in 1400

\section{Price
5 lbs $11 \mathrm{~b}$, \\ 425100}

425100

425100

425100

$600 \quad 140$

$750 \quad 175$

$\begin{array}{llll}6 & 50 & 1 & 40\end{array}$

$650 \quad 140$

$650 \quad 140$

$650 \quad 140$

$650 \quad 140$

$650 \quad 140$

$650 \quad 140$

$650 \quad 140$

$650 \quad 140$

$650 \quad 140$

$650 \quad 110$

1125250

$400 \quad 90$

405 90

$550 \quad 125$

500110

550125

$8001 \%$

$400 \quad 90$

$\leq 50 \quad 100$

reet Mountain

400 go Chinese Giant..

4 CO 80 Gulden Damn:

5 50. 125 L 2 . Bell cr B'11 Nose

600140 Rubs King......

40090 Cayenne........

450100 Jeapoiitan......

$550 \quad 125$

600125

900200

$500 \quad 125$

30065

$300 \quad 75$

30075

$800 \quad 175$

$300 \quad 65$

$300 \quad 75$

$450 \quad 110$

$350 \quad 80$

450100

$300 \quad 75$

$\begin{array}{lll}5 & 00 & 125\end{array}$

325

300

300

$300 \quad \% 5$

$800 \quad 1 \% 5$

800200

$300 \quad 75$

$300 \quad 75$

$300 \quad 75$

$300 \quad 75$

$300 \quad 75$

$400 \quad 90$

$\begin{array}{lll}5 & 00 & 125\end{array}$

809175

800175

Icicle ........... 550
PUIPKIN 10 lbs 5 lbs 1 lo.

Small Sugar... 6.50 $\leqslant 00100$

Lentucks Field $400 \quad 255$

Common Fiela.. $400 \quad 225 \quad 50$

Large Cheese ... 400 2 $25 \quad 50$

Niammoth Prizeiz ov 350150

Mam. Tours...1n 09 b $501=0$

King of JIasz... 12 $60 \quad 6 \% 3150$

RADIEF

Rusy Gem .

Es. Scar.Tumnip 5 ह0

Ruuna Dir Reù- 5 है

L.gscar.sn. Rip 5 aे

French frasia $:$ a 50

White Vienna.. E0

Wh. Sirasburg. 500

Chartier........ . 550

E5. Th. Turaip.5 59

Mixed ............ 5 हैil

LgBiackSpenisl 550

Chinese Rose.... 550

Scarlet Glude.... 559

Scar.Tur. Th.Til 550

Cincinvatti IF' $k+55$

Brs'sB't'Em Ali1200

Crimson Giant.- 550 SQUASH

WV Sum. Crookn' 850

325

325

(2)

$3.52 \quad-5$

325 i5

5 25 -

32575

$325 \%$

$325 \%$

3จะก

$325 \quad 75$

उ $25 \%$

$325 \%$

$3 \% 5$

$325 \% 75$

$3 \therefore 5$

$700 \quad 150$

$325 \quad 75$

$500 \quad 110$

5 GJ 110

NOTICE-We can make special prices on large amounts of seed, sets, bulbs, trees, hedges, insecticides fertilizer, etc. 


\section{WHOLESALE PRICES, FOR JANUARY 1930}

\section{ALL PRICES INCLUDE BAGS FREE HARDY ALFALFA}

Star Brand, N. W. Mountain Gr., U. S. Verified.\$9.90

Planet Brand, N.W. Mountain Gr., U. S. Verified 11.40 Dealers Fancy, N.W. Mtn. Gr., U. S. Verified 12.60 World Brand N. W. Mountain Gr., U. S. Verified 13.j0 World B. Kansas or Colorado Gr., U. S. Vfd.. . 12.90 Dakota Nंo. 12 World Brand, U. S. Verified.... 15.60 Montana Grown, World Brand, U. S. Verified.. 15.00 Grimn, Verified, World Brand, sealed bags.... 19.50 Grimm, Idaho, state sealed, $981 / 2 \%$ pure....... 18.60 Blackfoot Grimm, Certified, state sealed bags.. 22.50 Grimm, Idaho S't. S'l'd (Blkft. Section) $991 / 2 \%$ p. 21.00 Cossack, World Brand, Verified............ 20.00 HARDY, HOME GROWN, MEDIUM RED CLOVER Star Brand (Prime)......................... 10.50 Planet Brand (Extra Choice)................. 11.70

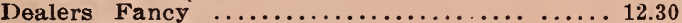

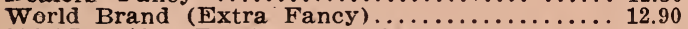
HARDY, HOME GROWN, MAMMOTH RED CLOVER

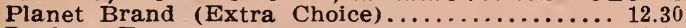

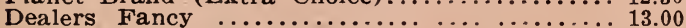
World Brand (Extra Fancy) .............. 13.50 HARDY, HOME GROWN, ALSIKE AND OTHER CLOVER Alsike Clover (Dealers Fancy).................... 11.70

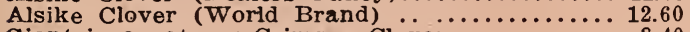
Giant incarnate or Crimson Clover........... 8.40

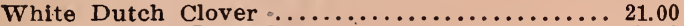

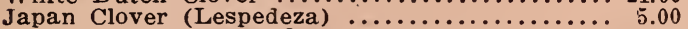
Korean Clover (Lespedeza) ............... 9.50

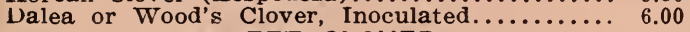
SWEET CLOVER

Fancy White Blossom, unhulled, $30 \mathrm{lb}$. bu...... 1.90 Prime White Blossom, hulled, $60 \mathrm{lb}$. bu. ........ 3.90 Choice White Blossom, hulled, 60 ib. bu...... 4.50 Fancy White Blossom, hulled, $60 \mathrm{lb}$. bu........ 5.00 Extra Fancy White Blossom, hulled, $60 \mathrm{lb}$. bu.. 5.40 Extra Fancy Grundy County White, 60 lb. bu.. 5.40 Choice Yellow Biennial, 60 lb. bu............. 4.70 Extra Fancy Yellow Biennial, $60 \mathrm{lb}$ bu............... 5.70 Yellow Annual (Fancy) $60 \mathrm{lb}$. bu............. 4.50 Hubam, Annual White (Extra Fcy.) 60 ib. bu.. 11.70 TIMOTHY \& CLOVER AND TIMOTHY MIXTURES Timothy (Star Brand) $\ldots \ldots \ldots \ldots \ldots \ldots \ldots \ldots \ldots .2 .50$

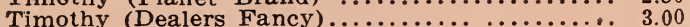

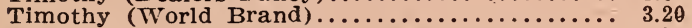
Alsike \& Timothy Mixed, No. 2, 45 lb. bu.... 3.90 Alsike \& Timothy Mixed, No. 1, 45 lb. bu..... 4.50 Red Clover \& Timothy Mixed, 45 1b. bu....... 4.30 Red Clover, Alsike \& Timothy Mxd., 45 ib. bu. 4.30 Alfalfa, Clover, Alsike \& Tim. Mxd., 45 lb. bu. 4.50 Sweet Clover and Timethy Mixed, $50 \mathrm{lb}$. bu... 4.00 Marvelous Mixture, $50 \mathrm{lb}$. bu............... 6.00 GRASSES AND OTHER HAY AND PASTURE CROPS

Fancy Kentucky Blue Grass............... 23.00

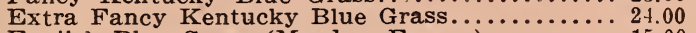

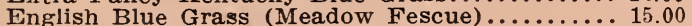

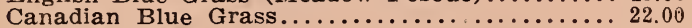

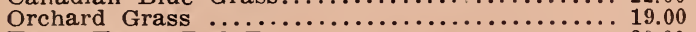

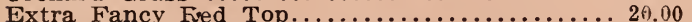

Bromus Inermus or Brome Grass............ 19.00

English and Italian Rye Grass.....................

Pasture or Meadow Mixture................... 15.00 Western Fye Grass....

Soy Beans, Black Eyebrow, In॰culated......... 3.10

Soy Beans, Dunfield and Ito San Inoculated.... 2.70

Soy Beans, Midwest, Inoculated .............. 2.70

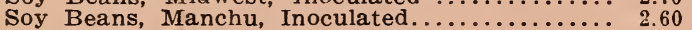

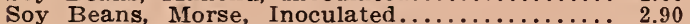

S'oy Beans, Virginia, Inoculated............ 3.20

Soy Beans, Black Early Wilson, Inoculated.... 3.50

Cowpeas, Whippoorwill ................ 3.60

Cowpeas; New Era ....................... 3.50

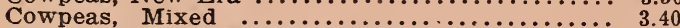

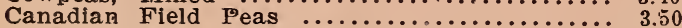

Sand, Winter or Hairy Vetch................. 5.50

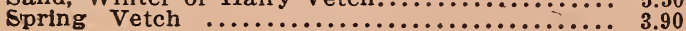

\section{CLARINDA}

$1 / 2$ bu. $1 / 4$ bu. $5.00 \$ 2.6$

$5.80 \quad 3.00$

$6.40 \quad 3.30$

$6.80 \quad 350$

$6.50 \quad 3.30$

$7.90 \quad 4.00$

7.60 . 3.90

9.80

9.40

11.30

10.60

10.10

5.30

5.90

6.20

6.50

6.20

6.60

6.80

\subsection{0}

5.90

6.40

4.30

10.60

2.60

4.80

3.10

1.00

2.00

2.30

2.60

2.80

2.80

2.40

2.90

2.30

5.90

1.30

1.50

1.60

1.70

2.00

2.30

2.20

2.20

2.30

2.10

3.10

\section{Ibs.}

11.60

12.10

7.60

11.10

9.60

10.10

9.60

7.10

7.60

6.10

per

bu.

3.20

2.80

2.70

3.00

3.30

3.60

3.70

3.60
3.50

3.60

5.60

4.00

5.70

5.40

5.10

2.70

3.00

3.20
3.30

3.20

3. 40

3.50

2.80

3.00

3.30

2.20

5.40

1.40

2.50

1.60

.60

1.10
1.20

1.40

1.50

1.50

1.30

1.50

1.20
3.00

.70

.80
.90

1.00

1.10

1.20

1.20

1.20

1.20

1.10

Ibs.

6.10

3.90

5.60

4.90

5.10

F. O.

Bu.

$\$ 10.15$

11.65

12.85

13.75

13.15

15.85

15.25

19.75

18.85

22.75

21.25
20.25

20.25

10.75

12.55

13.15

12.55

13.25

13.75

11.05

11.95
12.85

12.85
8.45

21.25

5.15

9.65

6.25

2.05

4.05

5.15

5.55

5.55

4.85
5.85

4.65

11.85

2.65

2.95

3.15

3.35

4.05

4.65

4.45

4.45

4.65

4.15

6.15

. CHICAGO

$\$ 5.15 \quad \$ 2.65$

5.95

6.55

6.95

6.65

8.05

7.75

9.95

9.55

11.45

10.75

5.45

6.05

6.35

6.65

6.35

6.75

6.95

\subsection{5}

6.05
6.55

4.35

10.75

2.65

4.85

3.25

1.05

2.05

2.65

2.85

2.85

2.45

2.95

2.35

5.95

1.35

1.55

1.65

1.75

2.35

2.25

2.25

2.35

2.15

3.15

100 lbs. 


$\begin{array}{rr}1 / 2 \text { bu. } & 1 / 4 \text { bu. } \\ 4.50 & 2.50 \\ 1.80 & 1.00 \\ 1.80 & 1.00 \\ 1.70 & .90 \\ 1.60 & .90 \\ 1.80 & 1.00 \\ 1.70 & .90 \\ 1.80 & 1.00 \\ 1.90 & 1.00 \\ 1.70 & .90 \\ 1.60 & .90 \\ 1.80 & 1.00 \\ 1.80 & 1.00 \\ 1.80 & 1.00 \\ 1.80 & 1.00 \\ 1.80 & 1.00\end{array}$

$\begin{array}{lll}\text { Bu. } & 1 / 2 \text { bu. } \quad 1 / 4 \text { bu. } \\ 8.65 & 4.55 & 2.55\end{array}$

Clarinda's Big Perfection.

Krug's Dent and Pride of Iowa.............. 3.50

Legal Tender and Improve

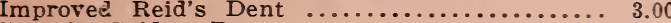

Berry's Golden Dent..................... 3.40

Iowa Gold Mine and White Cap Yellow Dent.. 3.20

Up-to-1.)ate Calico ....................... 3.40

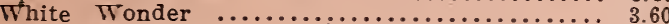

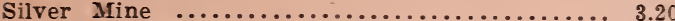

St. Charles Wkite ............................ 3.00

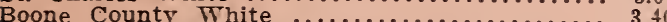

Pride of the North and Early Yellow Dent.... 3.40

Golden Cap and White Cap Red Dent........... 3.40

Minnesota No. 13 and Minnesota King........ 3.40

Longfellow and Silver Triumph Flint. crate; 5 crates for $\$ 22.50$

$1.55-2.55$

$\begin{array}{ll}1.85 & 1.05 \\ 1.85 & 1.05\end{array}$

$1.75 \quad .95$

$1.65 \quad .95$

1.851 .05

$\begin{array}{lr}1.75 & .95 \\ 1.85 & 1.05\end{array}$

$1.95 \quad 1.05$

$1.75 \quad .95$

$1.65 \quad .95$

$1.85 \quad 1.05$

$1.85 \quad 1.05$

$1.85-1.05$

$\begin{array}{ll}1.85 & 1.05 \\ 1.85 & 1.05\end{array}$
All Corn $25 \mathrm{c}$ per bushel less on 10 bushel lots. Guaranteed $98 \%$ or SEED OATS

Golden Rust Proof.

Montana Grown Regenerated Swedish Së.....

Fulghum (Select Iowa Grown) ................

New Alberta

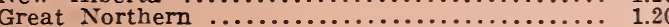

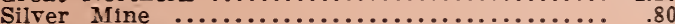

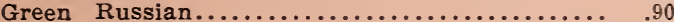

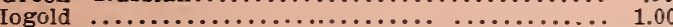

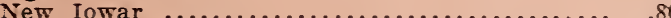

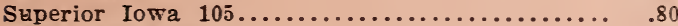

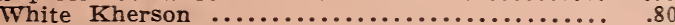

Early Champion ................................ 80

New Hulless Oats (Copper Treated)........... 2.00

10 bu.

BARLEY $10 \mathrm{bu}$.

Champion Beardless

1.30

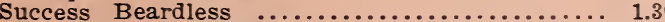

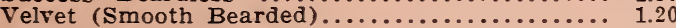

Improved White Hulless (copper treated)...... 1.9v

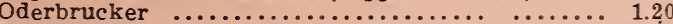

WHEAT, SPELTZ, SPRING RYE AND FïAX

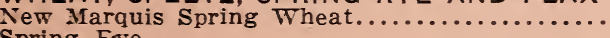

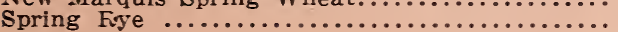

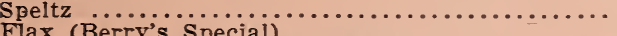

Flax (Berry's Special) $\ldots$ FORAGE

Millet, Golden Choice or German............ 1.50

Millet, Japanese (3j lbs. to the bushel)............. 1.80

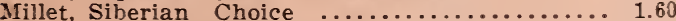

Millet, Broom Corn or $\mathrm{Hog}$ (or Proso)............ 1.60

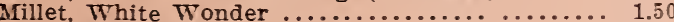

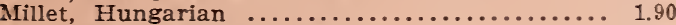

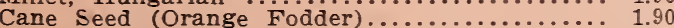

Cane Seed (Amber Fodder).................. 1.90

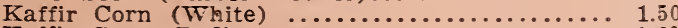

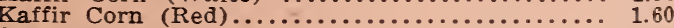

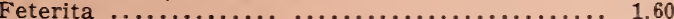

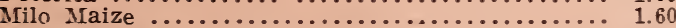

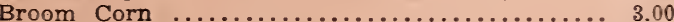

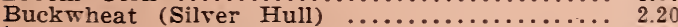

Buckwheat (Japanese) $\ldots \ldots \ldots \ldots \ldots \ldots \ldots \ldots \ldots \ldots \ldots \ldots \ldots \ldots .20$

Sudan Grass ......................... 7.00

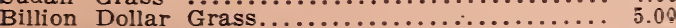

Dwarf Essex Rape

Berry's Annual Pork Producer.................. 3.50

Rape and Millet Mixed................... 5.75 POTATOES

per bu.

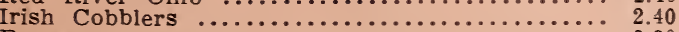

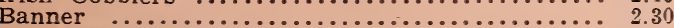

Rural New Yorker....................... 2.30

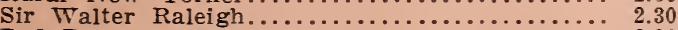

Red Rose

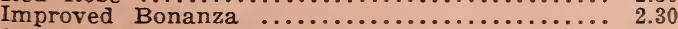

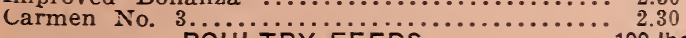

Planet Brand Chick Food........

100 lbs.

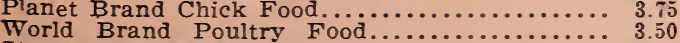

Star Brand Egg Food.................... 4.00

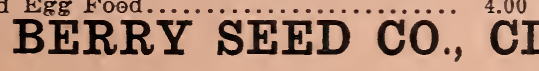

Ear Corn shipped from Clarinda only ermination per bu. bu

$1.10 \quad 1.20$

1.30

1.10

1.30

1.30

.90
1.00

1.10

.90

.90
.90

.90

2.10

per

bu.

1.40

1.40

1.30

2.00

1.30

1.30

1.40

1.20

1.40

1.40
1.00

1.10
1.20

1.00

1.00

1.00

1.00

2.20

bu.

.80

.80
.70

1.10

.70
.70

per bu. perbu.

$1.05 \quad 1.15$

1.25

1.05

1.35

1.25

1.25

1.35

1.35

.95

1.05

1.05

1.15

.82

.85

.85

2.15

10 bu.

per bu.

1.45

1.45
1.35

2.05

1.35

1.35

.95

.95

2.25

per

bu.

1.55

1.55

1.45

2.15

1.45
1.45

per

1.25

1.45

1.25

1.45

1.45

1.05

1.15

1.25

1.05

1.05

1.05

2.35

2.10

1.10

2.15

2.25

1.85

1.20

1.20
5.10

1.25

1.95

1.35

5.10

1.15

2.70

1.75

1.60

1.90

1.70

1.70

1.60

2.00

2.00

2.00

1.60

1.70

1.70

1.70

3.10

2.30

2.30

$50 \mathrm{lbs}$.

3.60
2.60

4.10

1.80

3.00

per

bu.

2.50

2.40

2.40

2.40

2.40

2.40

2.40

.90

1.00

1.65

1.95

1.75

1.75

.90

1.10

1.10

1.10

2.05

2.05

2.25

1.65

1.75

1.75

3.15

2.35

1.20

1.20

25 Ibs.

1.90

1.40

2.10

1.00

1.60

bu.

1.30

1. 00

1.30

1.30

1.30

1.30

1.30

50 ibs. 5 lbs.

2. 05

1.85

1.85

1.75

2.15

2.15

2.15
1.75

1.75
1.85

1.85

1.85

3.25

2.45

00 .

7.25

5.25

8.25

3.75

6.00

10 bu.

per bu.

2.55

2.55

2.45 


\begin{tabular}{|c|c|c|c|c|c|c|c|c|c|}
\hline \multirow{2}{*}{$\begin{array}{c}\text { VARIRTY } \\
\text { SQUASH }\end{array}$} & \multicolumn{3}{|c|}{ PRICE } & VARIETY & \multicolumn{2}{|c|}{ PRICI } & VARIETY & \multicolumn{2}{|c|}{ PRICE } \\
\hline & Con & & & TOMATO $10 \mathrm{lbs}$. & $5 \mathrm{lbs}$ & $1 \mathrm{lb}$. & Marglobe.... 4000 & 2250 & 503 \\
\hline Ey. White Bush & 850 & 500 & 110 & Early Mich.....25 00 & 1375 & 300 & Early Jur.e_ 2750 & I5 00 & 325 \\
\hline Yel, Bush Scal.. & 850 & 500 & 110 & New Stone...... 1750 & 1000 & 225 & Jn. Baer..... 2250 & 1250 & 273 \\
\hline Chi. War. Hub. . & 850 & 500 & 110 & Acme $\ldots \ldots . . . .2500$ & 1375 & 300 & F'ldsE.Rd.H.50 00 & 2525 & 550 \\
\hline Sibley & 1200 & 700 & 150 & Beauty & 1250 & 275 & TURNIP & & \\
\hline $\begin{array}{l}\text { Golden Hub...- } 1 \\
\text { Boston Marrow } 1\end{array}$ & 1000 & 600 & 125 & Chalk's Ey.Jew'20 00 & 1225 & 250 & Pur.Tp.StL'f 450 & 250 & 60 \\
\hline & $\begin{array}{ll}10 & 00 \\
6 & 00\end{array}$ & $\begin{array}{l}550 \\
900\end{array}$ & $\begin{array}{ll}1 & 25 \\
200 & 00\end{array}$ & $\begin{array}{l}\text { Ponderosa._._. } 3500 \\
\text { Dw'f Champion } 2500\end{array}$ & $\begin{array}{l}1875 \\
1375\end{array}$ & $\begin{array}{l}400 \\
300\end{array}$ & White Egg... 600 & 350 & 75 \\
\hline $\begin{array}{l}\text { Banana } \\
\text { Mam. Chili..... } 1\end{array}$ & $\begin{array}{l}1600 \\
1400\end{array}$ & 750 & 175 & Yellow Plum.. 3000 & 1625 & 350 & Sweet Germ'n 600 & 350 & 75 \\
\hline Faxon $\ldots . . . .1$ & 1120 & 650 & 140 & Red Pear ...... 3000 & 1625 & 350 & Pur. T. Milan 900 & 560 & 110 \\
\hline Imp Hubbard. & 850 & 500 & 110 & Matchless_......2 2J 00 & 1125 & 250 & White Milan_11 \&? & 600 & 140 \\
\hline SPINACE & & & & Berry's Tree_.. 2500 & 1375 & 300 & White Globe_.5 00 & $2 \pi 5$ & 60 \\
\hline Bloomsdale..... & -450 & 250 & & Golden Queen. 3500 & 1875 & 400 & Cowhorn. & $2 \pi 5$ & 60 \\
\hline & 450 & 250 & 60 & Earlibell....... 3000 & 1625 & 350 & Purp, T.Globe 500 & $2: 5$ & 60 \\
\hline Long Standing & 450 & 250 & 60 & $\mathrm{~B} y^{\prime} \mathrm{s}$ Es. Wond. $5 j 00$ & 2875 & $6 \mathrm{co}$ & MIonar.R.Baga5 00 & 275 & 60 \\
\hline King of Den..... & & 250 & 60 & Sp'k's Ear ian'. 2250 & 1250 & $2 \pi$ & P.T.Ruta Baga5 00 & 275 & 60 \\
\hline
\end{tabular}

Following Prices Not Prepaid

BEANS CWt 30lbs $10 \mathrm{lbs}$ Pinto ............. $1600 \quad 510 \quad 180$

\begin{tabular}{|c|c|c|c|c|c|c|c|c|}
\hline BEANS & & & & & & PEAS Y S S & sulos & \\
\hline New Br. Wax. 2500 & 780 & 270 & White Wonder...1000 & 510 & 180 & Little Marvel _.18 00 & 570 & 200 \\
\hline Imp. Blk Wax 2200 & 690 & 240 & White Navy _......1500 & 480 & $1 \% 0$ & First and Best..15 00 & & 170 \\
\hline Davis' wh Wax 2500 & 780 & $2 \div 0$ & SWEET CORN cWt & $30 \mathrm{lbs}$ & $10 \mathrm{lbs}$ & A mer Wonder. 1600 & 510 & \\
\hline Wa'wel'sK Wx 2500 & 780 & $2 \pi 0$ & Early Wh. Cory _. 1000 & 330 & 120 & Nott's Excelsi'r 1700 & 540 & 190 \\
\hline olden $\operatorname{Tax} \ldots 2500$ & 780 & 270 & Earls Minnesota.10 00 & 330 & 120 & Premlum Gem. 1800 & 570 & \\
\hline Mam. Wh.Pod_28 00 & 870 & 300 & Howling Mob.... 1000 & 330 & 120 & Alaska..........1600 & 510 & \\
\hline few Bountiful 3) 00 & 930 & 320 & Co. Gentlem n... 1500 & 480 & 170 & Stratagem. & & \\
\hline t St'gless G.P 3300 & 1020 & 350 & Stowell's Evergr' 1200 & 390 & & udance.-. & & \\
\hline Str'gl'ss Gr'n P 3300 & 1020 & 350 & Golden Bantam_.1200 & 390 & 140 & Champ.of Eng. 1800 & & \\
\hline fugee $1000-1.3300$ & 1020 & 350 & Ey Evergreen....1200 & 390 & 140 & Wh. Marrowfat 1500 & & \\
\hline 5. Valentine. 2200 & 680 & 240 & Peep O Das & 390 & 140 & Telephone_---1800 & 570 & \\
\hline nd'B'sh Lim. 1900 & 600 & 210 & Earìy Sensation - 1500 & $(48)$ & $17 \mathrm{~J}$ & $\begin{array}{l}\text { Everbearing ... } 1900 \\
\text { Advancer.... } 1800\end{array}$ & $\begin{array}{l}660 \\
5 \% 0\end{array}$ & \\
\hline rdb'kB. Lim.35 00 & 1080 & $3 \pi 0$ & Late Niammoth-1200 & $\begin{array}{l}390 \\
480\end{array}$ & 170 & Gradus ........... 1700 & 540 & 190 \\
\hline Bur.Bush Lim. 2500 & 780 & 270 & ZigZag Evergr'n_1200 & $\begin{array}{l}480 \\
390\end{array}$ & 140 & Potaltch 1960 & & \\
\hline Goldn Clust. 2200 & 680 & 240 & Kendall's Giant_-1200 & $\begin{array}{l}390 \\
390\end{array}$ & & Pedigree Ex.Es 1800 & & \\
\hline Ken't. Wonder_18 00 & 570 & 200 & Early Sunshine.. 1800 & $5 \% 0$ & 200 & Tom Thumb_-- 16 & & \\
\hline zy Wife. & 690 & 240 & POP CORN 100Ibs & $30 \mathrm{lbs}$. & $10 \mathrm{lbs}$ & $\begin{array}{l}\text { ThomasLaxton } 1900 \\
\text { Iam.Luci's Sug } 1800\end{array}$ & $\begin{array}{l}6 \text { co } \\
570\end{array}$ & \\
\hline & 699 & 240 & Rice [seeâ] 1000 & 33 & & Alderman....... 1700 & & \\
\hline den 2100 & 660 & 230 & n's Golden $-10 \mathrm{co}$ & & & Surprise $\ldots \ldots,-\ldots, 18$ & & \\
\hline Ky. Won. Wax 2200 & 690 & 240 & Tananase Hul-less 110 & 3 *i) & & Sunt ton's Pione'r $18 \mathrm{n}$ & & 203 \\
\hline Cut Short_..... 2200 & 690 & 240 & Dynamite......... 150 & $48)$ & $1 \% 0$ & New Laxtonian 1900 & $6 i 0$ & 210 \\
\hline
\end{tabular}

\begin{tabular}{|c|c|c|c|c|c|c|c|c|c|}
\hline STRAWBERRY & 200 & 500 & 1000 & 5000 & 10000 & CABBAGE PLANTS & 1000 & 5000 & 10000 \\
\hline Senator Dunlap & 150 & 325 & 600 & $25 \mathrm{co}$ & 4500 & Norseman................ & 225 & & \\
\hline Gibson & & 325 & 600 & 2500 & 450 & Early Jersey Wakefield. & & & 1500 \\
\hline Dr. Burrill _......- & 150 & 325 & 600 & 2500 & $45 \mathrm{co}$ & $\begin{array}{l}\text { Earls All Head } \\
\text { Copenhagen Mlarke }\end{array}$ & & $\begin{array}{l}8 \pi \\
8 \% 5\end{array}$ & 1500 \\
\hline & & & $\begin{array}{l}4000 \\
1500\end{array}$ & $15 ? 00$ & $\begin{array}{l}200<0 \\
100<0\end{array}$ & Earls & & 875 & 1500 \\
\hline $\begin{array}{l}\text { Imp. Prcgressive } \\
\text { Champ on........ }\end{array}$ & & $\begin{array}{l}860 \\
800\end{array}$ & $\begin{array}{l}1500 \\
1500\end{array}$ & $\begin{array}{ll}6000 \\
60 & 00\end{array}$ & $100 \mathrm{CO}$ & ONION SETS & 50 liss & 25 Ibs & $10 \mathrm{lbs}$ \\
\hline Mastodon........ & 500 & 10 & $18 \mathrm{co}$ & 8500 & $150 \mathrm{CO}$ & Red & & & \\
\hline Asparagus Roots & $1 \mathrm{r} r$. & 450 & 800 & 3500 & 600.5 & Bottom & & & \\
\hline Rhubarb Roots & $2 Y_{i}$ & 650 & 120 & 6000 & 9000 & $\begin{array}{l}\text { E enpzer } \\
\text { White Bottom...... } 13 \text { c0 }\end{array}$ & $\begin{array}{l}600 \\
700\end{array}$ & & $\begin{array}{ll}1 & 40 \\
1 & \text { f } 0\end{array}$ \\
\hline $\begin{array}{l}\text { Knubarb Koots... } \\
\text { Hot Kaps-paper }\end{array}$ & 900 & 2000 & 3500 & & & Winter Top........ 800 & & 250 & 110 \\
\hline & lant $\mathrm{c}$ & overs & 1150 & 5500 & 10750 & \multicolumn{2}{|c|}{ Onion Plants-6000 per crate 495} & \multicolumn{2}{|c|}{5 crates $20 \mathrm{co}$} \\
\hline All Stancard Ve & arieti & & 50 & 100 & 1000 & SPIREA & 100 & 1000 & \\
\hline Apple Trees Miail & S'ze. & & -1000 & 1300 & 1500 & ch.-.-. 800 & $\begin{array}{l}1500 \\
2000\end{array}$ & $\begin{array}{l}12500 \\
1 ; 500\end{array}$ & \\
\hline " " Exp. & S.ze... & & & & & $-0-11$ & & & \\
\hline$" \quad$ "Extra & a Larg & e. & .2500 & 4000 & 35000 & $\ldots 1800$ & & 275 & \\
\hline Cherry Trees Mail & 1 Size & & .1500 & 2500 & 22500 & AMOOR PRIVET & & & \\
\hline & & & & 4500 & 37500 & & & & \\
\hline $\begin{array}{l}\text { Peach Trees Mail } \\
\text { Exp. }\end{array}$ & $\begin{array}{l}\text { Size } \\
\text { Size. }\end{array}$ & & $\begin{array}{l}10<0 \\
2000\end{array}$ & $\begin{array}{l}1800 \\
3500\end{array}$ & $\begin{array}{l}15000 \\
30000\end{array}$ & $\begin{array}{l}2-3 \text { feet } \\
3-4 \text { feet }\end{array}$ & $\begin{array}{l}1300 \\
1400\end{array}$ & $\begin{array}{l}100 \\
120\end{array}$ & \\
\hline
\end{tabular}

All Standard varieties of Fruit Trees, Grapes, Shrubbery, Roses, Evergreens, or Shade Trees not found in our Catalogue can be furnished approximately at same price as in our Catalogue. 